\title{
Nanoscale organization of the endogenous ASC speck
}

Ivo M. Glück ${ }^{1,2}$, Grusha Primal Mathias ${ }^{1,3}$, Sebastian Strauss ${ }^{4,5}$, Thomas S. Ebert ${ }^{6,7}$, Che Stafford ${ }^{6,7}$, Ganesh Agam $^{1,2}$, Suliana Manley ${ }^{8}$, Veit Hornung ${ }^{6,7}$, Ralf Jungmann ${ }^{4,5}$, Christian Sieben ${ }^{8,},{ }^{*}$, Don C. Lamb ${ }^{1,2,{ }^{*}}$

1 Department of Chemistry, Ludwig Maximilians-Universität München, Butenandtstraße 5-13, 81377 München, Germany

2 Center for Nano Science (CENS), Ludwig Maximilians-Universität München, Butenandtstraße 5-13, 81377 München, Germany

3 Deutsches Zentrum für Neurodegenerative Erkrankungen (DZNE), Bonn, Germany und Universität Köln, Köln, Germany

4 Faculty of Physics and Center for Nanoscience, Ludwig Maximilian University, Munich, Germany

5 Max Planck Institute of Biochemistry, Martinsried, Germany

6 Gene Center, Ludwig-Maximilians-Universität, Munich, Germany

7 Department of Biochemistry, Ludwig-Maximilians-Universität, Munich, Germany

8 Laboratory of Experimental Biophysics, École Polytechnique Fédérale de Lausanne, BSP 427 (Cubotron UNIL), Rte de la Sorge, CH-1015 Lausanne, Switzerland

$\S$ Current address: Nanoscale Infection Biology, Helmholtz Centre for Infection Research, Inhoffenstr. 7, 38124 Braunschweig, Germany

\footnotetext{
* Correspondence should be addressed to

Christian Sieben, christian.sieben@helmholtz-hzi.de

or
}

Don C. Lamb, don.lamb@cup.uni-muenchen.de 


\begin{abstract}
The NLRP3 inflammasome is a central component of the innate immune system. Its activation leads to the formation of a supramolecular assembly of the inflammasome adaptor ASC, denoted as the "ASC speck". Different models of the overall structure of the ASC speck as well as the entire NLRP3 inflammasome have been reported in literature. While many experiments involve overexpression or in vitro reconstitution of recombinant ASC, the cytoplasmic endogenous ASC speck remains difficult to study due to its relatively small size and structural variability.

Here, we use a combination of fluorescence imaging techniques including dual-color 3D superresolution imaging (dSTORM and DNA-PAINT) to visualize the endogenous ASC speck following NLRP3 inflammasome activation. We observe that the complex varies in diameter between $\sim 800$ and $1000 \mathrm{~nm}$ and is composed of a dense core from which filaments reach out into the periphery. We used a combination of anti-ASC antibodies as well as a much smaller nanobody for labeling and show that the larger complexes do not reliably label the dense core whereas the nanobody, which has a lower binding affinity, is less efficient in labeling the lower-density periphery. Imaging whole cells using dSTORM, furthermore, allowed us to sort the imaged structures into a quasi-temporal sequence suggesting that the endogenous ASC speck becomes mainly denser but not much larger during its formation.
\end{abstract}




\section{Introduction}

Inflammasomes are large, multiprotein complexes that assemble upon activation of cellular pattern recognition receptors (PRRs) as part of the response of the innate immune system (1). For the NOD-, LRR- and pyrin domain-containing protein 3 (NLRP3) inflammasome, the key components include the NLRP3 sensor protein, the adaptor protein Apoptosis-associated speck-like protein containing a Caspase activation, and recruitment domain (ASC) (2) (3) and the inflammatory caspase 1 (CASP1) (4) (5) (6). NLRP3 activation triggers recruitment of ASC and CASP1 leading to a single micrometersized assembly. For the structure of ASC within the inflammasome, Masumoto et al. coined the term "ASC speck" (3) (7). ASC is composed of a Caspase activation and recruitment domain (CARD) (8) and a Pyrin domain (PYD) (9) connected by a semi-flexible linker. The individual domains of ASC have a high tendency for homotypic interactions due to their high complementarity in structure and charge, leading to the large, multiprotein, inflammasome assembly (10) (11) (12) (13). However, how the speck is organized is not yet fully understood and is the subject of intensive research.

ASC $(22 \mathrm{kDa})$ is soluble at low $\mathrm{pH}$ and in chaotropic solution but, at physiological $\mathrm{pH}$, the protein can assemble into filaments as has been shown in vitro by solid-state NMR spectroscopy and electron microscopy (EM) (2) (3) (14) (15) (16) (17) (18) (19). The ASC speck can also be purified from inflammasome-activated cells expressing ASC endogenously and was found to form a star-shaped assembly (7), isolated filaments or amorphous aggregates potentially composed of intertwined filaments (17) (20). In cells, ASC specks were visualized by microscopy using immunofluorescence or expression of ASC tagged with a fluorescent protein. Upon overexpression, the resulting speck was usually much larger than $1 \mu \mathrm{m}$ and occasionally showed filaments protruding from the edge of the structure (18) (20) (21) (22). In contrast, diffraction-limited immunofluorescence imaging of the endogenous ASC speck revealed a spot of about $1 \mu \mathrm{m}$ in diameter. Due to the above-mentioned propensity of ASC to self-assemble, it is unclear whether the endogenous structure resembles the complexes observed in vitro or upon ASC overexpression. Interestingly, one approach to labeling ASC using a GFP-labeled nanobody directed against the CARD domain lead to detection of an intermediate structure during speck formation of filamentous ASC but no ASC specks were observed (23). Higher resolution studies, either by EM (24) or super-resolution fluorescence microscopy (25), could resolve the endogenous speck as an amorphous aggregate potentially made up of intertwined filaments. A number of studies describe the ASC speck as a hollow, ring-shaped complex (3) (6) (21, 26) (27) (28) (29) (30) (31) (32) (33) (34) and, based on this observation, various models for ASC speck and inflammasome formation have been proposed (30) (31) (35) (36) (37) (38). Other studies show an amorphous speck assembly (17) (23) (39). Hence, despite its relevance for understanding inflammasome formation, the nanoscale organization of the endogenous ASC speck is not fully understood.

Here, we used quantitative widefield microscopy, confocal microscopy and single-molecule localization microscopy (i.e. 3D dual-color $\mathrm{dSTORM}$ and 3D DNA-PAINT) to investigate the structure of the endogenous ASC speck. Our data resolved filaments protruding from a dense core of the endogenous ASC speck supporting the amorphous nature of the complex. By using two complementary labelling approaches comparing antibody- with nanobody-labelled ASC, we could probe the organization of and density differences within the ASC speck. We found that nanobodies labelled the center of the speck while the antibody was predominantly detected in the periphery of the complex, occasionally exhibiting a hollow center. Furthermore, we analyzed the redistribution of ASC into the speck using a single-cell analysis, allowing us to sort specks with respect to the degree of ASC recruitment. Our results indicate that endogenous specks mainly become denser but not larger during inflammasome assembly. 


\section{Results}

\section{Endogenous specks vary strongly in ASC content}

We investigated the organization of the endogenous ASC speck in THP-1 cells. The cells were primed using lipopolysaccharide (LPS) (40) followed by stimulation with the bacterial toxin nigericin (41). After 90 minutes, the cells were fixed using paraformaldehyde and stained with a primary monoclonal antibody against ASC in combination with a secondary Alexa Fluor 647-tagged $\mathrm{F}_{\mathrm{ab}}$ fragment. To get a first insight into the distribution of ASC in the cell, we imaged the cells at low magnification $(10 \times)$ using confocal microscopy. In unstimulated cells, ASC was distributed throughout the cell, including the nucleus (Figure 1A, left panel). Upon NLRP3 inflammasome activation, ASC relocated into the characteristic single perinuclear speck in about 30\% of the cells (Figure 1A, right panel) (42). We could also observe extracellular specks as previously reported (20) (24) (Supplementary Figure S1). Next, we used high magnification (60x) widefield microscopy, which allowed us to study the speck morphology and to estimate the amount of incorporated ASC as measured by the signal of bound antibodies. We imaged 59 individual ASC specks, which appear as spherical complexes differing in size between approximately 0.5 and $1 \mu \mathrm{m}$ diameter (Figure 1B). Analysis of the integrated intensity revealed that the amount of incorporated ASC can vary by almost one order of magnitude (Figure 1C). The widefield microscopy images also confirmed our observations using confocal imaging that ASC is distributed throughout unstimulated cells (Figure 2A, left panel). As expected, for cells showing a speck, we observed an almost complete depletion of the cytoplasmic ASC and a single, bright ASC spot (Figure 2A, right panel). These observations are consistent with previous reports showing that the vast majority of cytoplasmic ASC becomes redistributed into the ASC speck upon activation of the NLRP3 inflammasome (43). 


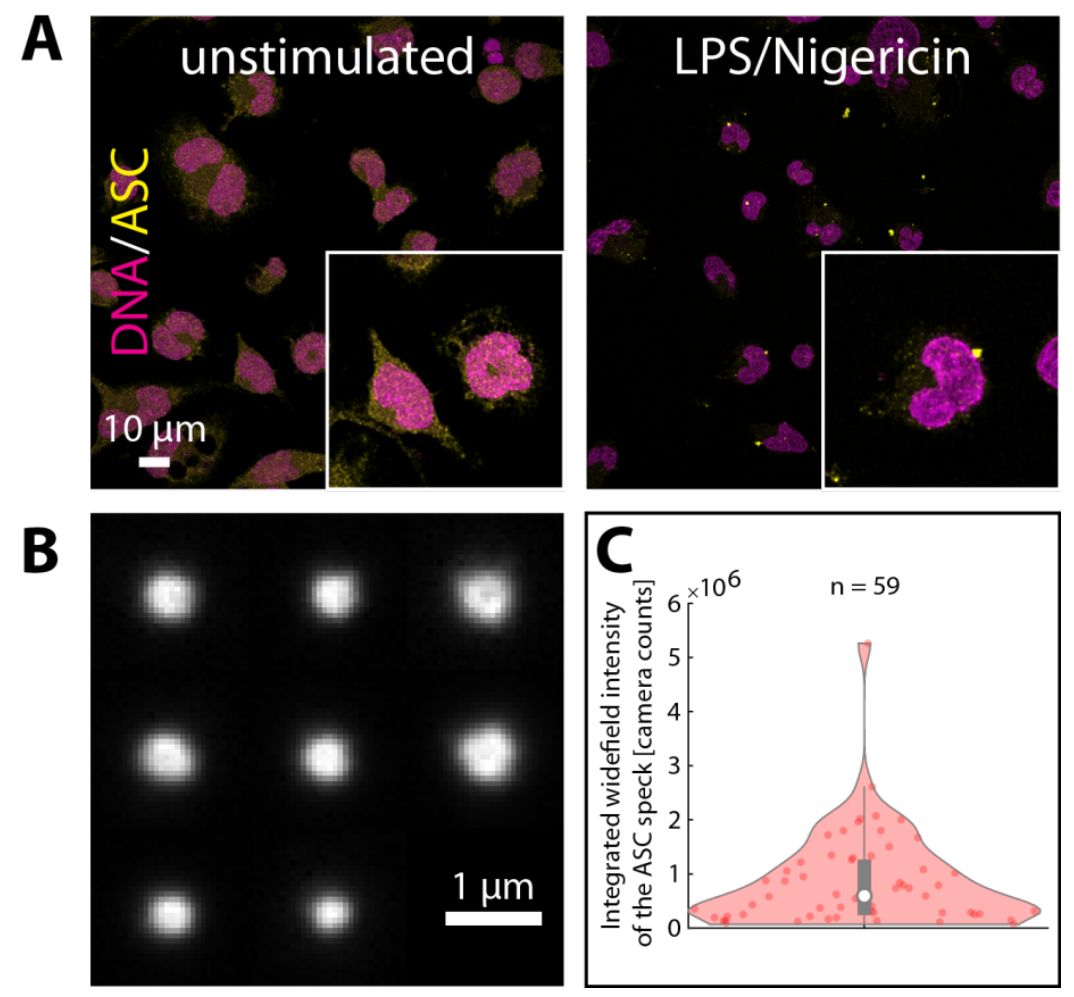

Figure 1 - LPS + Nigericin stimulation of THP-1 cells induces ASC speck formation of various physical sizes and amount of assimilated protein. A) Confocal images (maximum projections) of fluorescently labeled ASC (shown in yellow) in unstimulated (left panel) and in LPS + Nigericin-stimulated THP-1 cells (right panel). The formation of ASC specks after stimulation is clearly visible. DNA is stained using a DAPI reagent (shown in magenta). B) A montage of ASC specks immunostained using a primary antibody and a secondary Alexa Fluor 647-labeled $\mathrm{F}_{\mathrm{ab}}$ fragment and imaged by diffraction-limited widefield illumination with 60x magnification. The speck size varies and the appearance of a darker center is observed for some of the specks. C) A violin plot of the integrated widefield intensity of ASC specks imaged as described for panel B. There is a large variation in the intensity, which serves as an indicator for the amount of protein incorporated into the speck.

Non-speck incorporated ASC clusters decrease in number but maintain a similar size distribution during ASC speck formation

To study the distribution of ASC on the nanoscale, we used direct stochastic optical reconstruction microscopy (dSTORM) (44), which was previously utilized to study the nanoscale organization of the human centriole (45). dSTORM reconstructions confirmed the cytoplasmic ASC distribution in unstimulated cells and its redistribution into a single speck after inflammasome activation. Interestingly, the increased detection sensitivity of dSTORM allowed us to visualize a non-speckbound ASC population in the cytoplasm of activated cells (Figure 2B). We performed a density-based clustering analysis to quantify the cytosolic (non-speck) population of ASC (Supplementary Figure S2 and Supplementary Figure S3). Using a Density-Based Spatial Clustering of Applications with Noise (DBSCAN) analysis (46) (Figure 2C), we observed a decrease in the cluster density from an average of 0.40 clusters per $\mu \mathrm{m}^{2}$ in unstimulated cells to 0.18 clusters per $\mu \mathrm{m}^{2}$ in cells showing a speck (Figure 2D). This is consistent with the observed ASC recruitment (Figure 2A). Next, we investigated the number of localizations per cluster and the cluster size by calculating the radius of gyration $\left(R_{g}\right)$. We found the size of non-speck incorporated clusters to be very similar between unstimulated and stimulated cells $\left(R_{g}<20 \mathrm{~nm}+\right.$ localizations/cluster $\left.<60\right)$ (Figure 2E, F). However, in speck containing cells, an additional cytoplasmic cluster population appears with a larger size and more localizations $\left(R_{g} 20-80 \mathrm{~nm}+\right.$ localizations/cluster $\left.>60\right)$. We also applied a Ripley's K clustering 
bioRxiv preprint doi: https://doi.org/10.1101/2021.09.17.460822; this version posted September 20, 2021. The copyright holder for this preprint (which was not certified by peer review) is the author/funder, who has granted bioRxiv a license to display the preprint in perpetuity. It is made available under aCC-BY-NC-ND 4.0 International license.

analysis (47) as an alternative approach for characterizing the spatial distribution non-speck incorporated ASC. The Ripley's K clustering analysis (Figure 2G) confirmed the decrease in cytoplasmic cluster density in cells containing a speck as shown by the shift of the obtained L(r)-r curve towards higher values and an increase of the $L(r)-r$ maximum value consistent with a small population of larger clusters. 

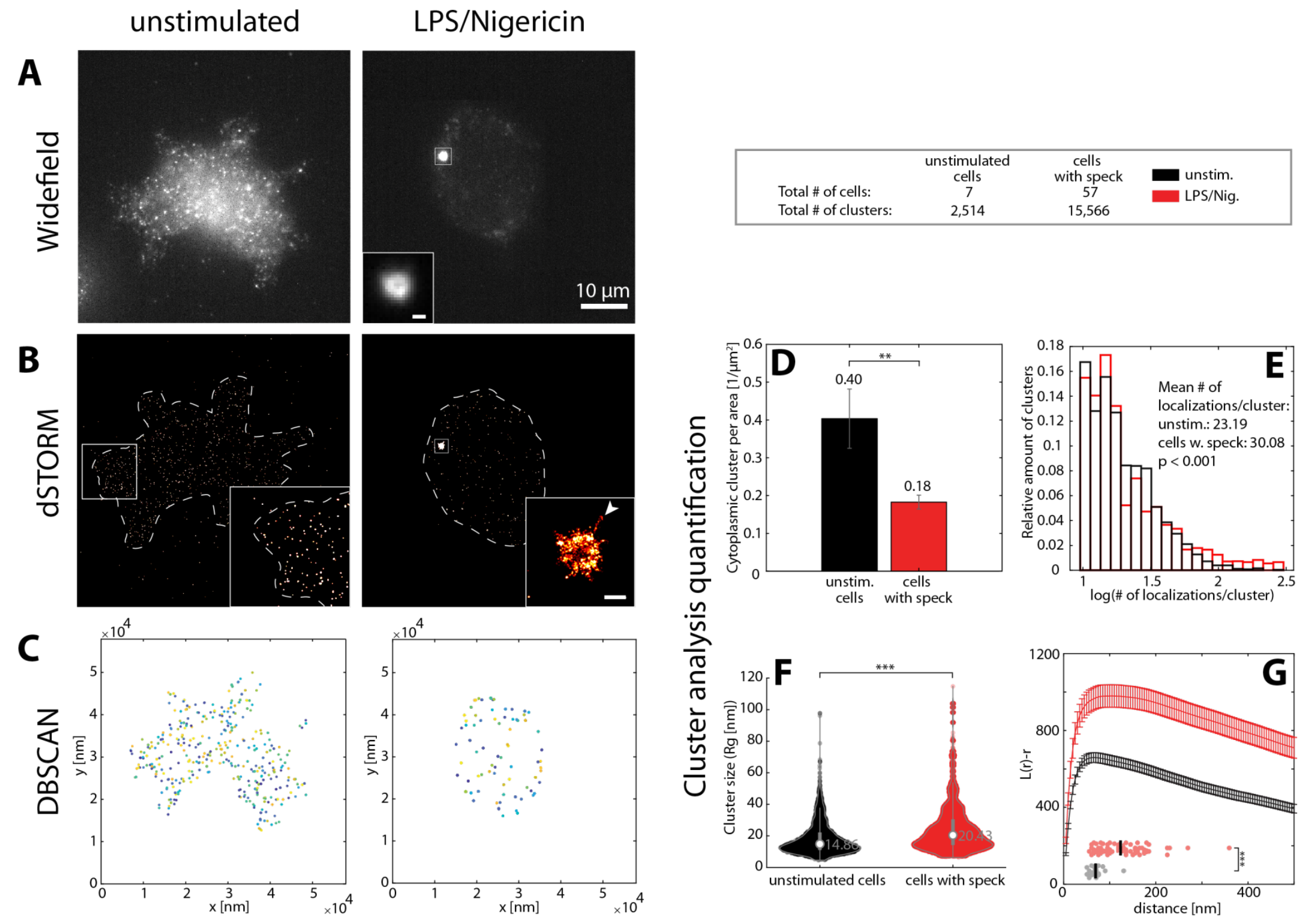

7 
bioRxiv preprint doi: https://doi.org/10.1101/2021.09.17.460822; this version posted September 20, 2021 . The copyright holder for this

preprint (which was not certified by peer review) is the author/funder, who has granted bioRxiv a license to display the preprint in perpetuity. It is made available under aCC-BY-NC-ND 4.0 International license.

Figure 2 - Whole-cell dSTORM super-resolution microscopy of endogenous ASC in THP-1 cells. A, B) The distribution of ASC in non-stimulated cells (left panels) and the redistribution of ASC into the speck after stimulation with LPS and Nigericin (right panels) observed using diffraction-limited widefield imaging (A) and ASTORM (B). dSTORM additionally resolves filaments protruding from the speck core (one example is highlighted by the arrow). Scale bars in insets correspond to $500 \mathrm{~nm}$. C) DBSCAN clustering of (non-speck bound) cytosolic ASC localizations confirms depletion of the protein from the cytosol after speck formation. Color coding is used to distinguish individual clusters. D - G) The results of a DBSCAN analysis are shown. D) The non-speck incorporated ASC cluster density decreases in cells exhibiting a speck due to recruitment of the protein into the speck. The error bars indicate the standard error of the mean calculated from the analysis results of individual cells. E), F) The distribution of the number of localizations (E) and violin plots of the radius of gyration $\left(R_{g}\right)(\mathrm{F})$ for unstimulated cells and cells forming a speck. The distributions are similar with an additional population observable in speck-producing cells with more localizations and a larger size compared to non-stimulated cells. White circles in the violins indicate the median of the distribution. The statistical significance in D-F was assessed by a Two-Sample Kolmogorov-Smirnov Test $\left(^{*}\right) \mathrm{p}<0.05,\left({ }^{* *}\right) \mathrm{p}<0.01,(* * *) \mathrm{p}<0.001$. G) A Ripley's K analysis of the ASC clusters. The Ripley's K function confirms the decrease in cluster density after speck formation as well as the increase in cluster size. The curves show the mean \pm SD of 40 cells imaged from three independent experiments. For the estimation of the curve's maximum (G, lower part), we measured at least 2 ROIs per cell (100 ROIs in total). Statistical significance was assessed by student's t-test $(* *) \mathrm{p}<0.005,(* * *) \mathrm{p}<0.001$. 
bioRxiv preprint doi: https://doi.org/10.1101/2021.09.17.460822; this version posted September $20,2021$. The copyright holder for this preprint (which was not certified by peer review) is the author/funder, who has granted bioRxiv a license to display the preprint in perpetuity. It is made available under aCC-BY-NC-ND 4.0 International license.

Table 1 - Summary of measurement parameters on unstimulated cells and LPS + Nigericin-treated cells containing an ASC speck *All values occurred just once; MAD: Median absolute deviation of the median

\begin{tabular}{|c|c|c|c|c|c|c|c|}
\hline & & $\begin{array}{l}\text { unstimulated } \\
\text { cells }\end{array}$ & \multicolumn{5}{|c|}{$\begin{array}{l}\text { LPS + Nigericin-treated, } \\
\text { Speck-containing cells }\end{array}$} \\
\hline & & $\begin{array}{c}\text { Primary } \\
\text { antibody }+ \\
\text { secondary } \\
\text { AF 647- } \\
\text { labeled } \mathrm{F}_{\mathrm{ab}} \\
\text { fragment }\end{array}$ & $\begin{array}{c}\text { Primary } \\
\text { antibody } \\
+ \\
\text { secondary } \\
\text { AF 647- } \\
\text { labeled } \\
F_{a b} \\
\text { fragment } \\
w+\text { w/o } \\
\text { additional } \\
\text { DL755- } \\
\text { labeled } \\
\text { nanobody }\end{array}$ & $\begin{array}{c}\text { Primary } \\
\text { antibody } \\
+ \\
\text { secondary } \\
\text { AF 647- } \\
\text { labeled } \\
\mathrm{F}_{\mathrm{ab}} \\
\text { fragment } \\
\text { only }\end{array}$ & $\begin{array}{c}\begin{array}{c}\text { Primary } \\
\text { antibody } \\
+\end{array} \\
\text { secondary } \\
\text { AF 647- } \\
\text { labeled } \\
\text { Fab }_{\text {ab }} \\
\text { fragment } \\
\text { W } \\
\text { additional } \\
\text { DL755- } \\
\text { labeled } \\
\text { nanobody }\end{array}$ & $\begin{array}{l}\text { AF 647- } \\
\text { labeled } \\
\text { nanobody } \\
\text { only }\end{array}$ & $\begin{array}{c}\text { DL 755- } \\
\text { labeled } \\
\text { nanobody } \\
\text { W } \\
\text { additional } \\
\text { primary } \\
\text { antibody } \\
+ \\
\text { secondary } \\
\text { AF 647- } \\
\text { labeled } \\
F_{\text {ab }} \\
\text { fragment } \\
\text { staining }\end{array}$ \\
\hline $\begin{array}{l}\text { \# of cells or } \\
\text { specks }\end{array}$ & & 7 & $\begin{array}{c}57 \\
\text { (cluster } \\
\text { analysis) } \\
60 \text { (speck } \\
\text { analysis) }\end{array}$ & 21 & 39 & 92 & 42 \\
\hline \# of clusters & & 2,514 & 15,566 & - & - & - & - \\
\hline $\begin{array}{l}\text { Mean } \\
\text { cytoplasmic } \\
\text { clusters/area } \\
{\left[1 / \mu \mathrm{m}^{2}\right]}\end{array}$ & & 0.18 & 0.40 & - & - & - & - \\
\hline $\begin{array}{l}\text { Mean \# of } \\
\text { localizations } \\
\text { per cluster }\end{array}$ & & 23.19 & 30.08 & - & - & - & - \\
\hline $\begin{array}{l}\text { Median } \\
\text { cluster size } \\
\left(R_{g}\right)[\mathrm{nm}]\end{array}$ & & 14.86 & 20.43 & - & - & - & - \\
\hline $\begin{array}{l}\text { Mode } \\
\text { cluster size } \\
\left(2 \mathrm{D}-R_{g}\right) \\
{[\mathrm{nm}]}\end{array}$ & & 6.04 & 7.05 & - & - & - & - \\
\hline \multirow{4}{*}{$\begin{array}{l}\text { xy } \\
\text { Eccentricity } \\
\text { of speck }\end{array}$} & Mean & - & 1.23 & 1.14 & 1.29 & 1.33 & 1.5 \\
\hline & Median & - & 1.19 & 1.13 & 1.24 & 1.23 & 1.4 \\
\hline & Mode & - & 1.02 & 1.02 & 1.04 & 1.07 & 1.03 \\
\hline & MAD & - & 0.08 & 0.04 & 0.07 & 0.11 & 0.19 \\
\hline \multirow{4}{*}{$\begin{array}{l}2 \mathrm{D}-R_{g} \text { of } \\
\text { speck }[\mathrm{nm}]\end{array}$} & Mean & - & 325.2 & 332.6 & 321.2 & 298.1 & 158.3 \\
\hline & Median & - & 321.3 & 324.0 & 319.7 & 281.9 & 133.9 \\
\hline & Mode & - & 80.4 & 80.4 & 242.1 & 310.6 & 19.9 \\
\hline & MAD & - & 30.9 & 35.9 & 29.0 & 36.5 & 49.9 \\
\hline \multirow{4}{*}{$\begin{array}{l}\text { Number of } \\
\text { localizations } \\
\text { per speck }\end{array}$} & Mean & - & 5010 & 7209 & 3826 & 5338 & 3848 \\
\hline & Median & - & 4113 & 7180 & 3307 & 2749 & 2787 \\
\hline & Mode & - & $\mathrm{N} / \mathrm{A}^{*}$ & 1062 & N/A* & 1297 & 345 \\
\hline & MAD & - & 1430 & 2691 & 954 & 1872 & 2061 \\
\hline
\end{tabular}


$\underline{\text { Super-resolution microscopy reveals distinct morphologies of the endogenous ASC speck }}$

Next, we investigated the nanoscale organization of the ASC speck itself (45) (48). We started by labelling endogenous ASC using a primary antibody in combination with a secondary $F_{a b}$ fragment as described above. A large proportion of the specks appeared as almost round amorphous structures with a diameter of about $1 \mu \mathrm{m}$ exhibiting a rough surface with short protrusions (Supplementary Figure S4A). Interestingly, for a large number of the specks, the higher resolution obtained by dSTORM imaging resolved ASC filaments reaching out from the dense core of the speck (Figure 3A). The number of clearly resolved filaments per structure varied but we rarely observed more than $\sim 10$. We measured the diameter of the filaments using a gaussian fitting of the intensity profile of multiple cross sections along each filament. The filament diameter was derived from the full width half maximum of the intensity profile, where we found a median value of $37.1 \mathrm{~nm}$ (Figure 3B). Considering the size of a primary/secondary $\mathrm{F}_{\mathrm{ab}}$ antibody complex $(\sim 11 \mathrm{~nm})$ (49) used for labeling, the measured value corresponds to an actual thickness of the filament of $\sim 15 \mathrm{~nm}$. When compared to values obtained for filaments formed by ASC in vitro and studied by EM $(16 \mathrm{~nm})(15)$, this value indicates that the majority of filaments are isolated single filaments.

To check the validity of the results, we also imaged the endogenous ASC specks using a different labeling strategy and super-resolution approach. We performed DNA-PAINT super-resolution microscopy (50) (51) using BlaER1 cells, transdifferentiated into monocytes/macrophages in which ASC was endogenously tagged with TagRFP (52) (53) (54). Using this alternative system, we could reproduce the observed morphology of the endogenous ASC speck including filaments and a dense core. The filaments strikingly, were much longer than the ones observed by dSTORM in THP-1 cells (Figure 3C and Supplementary Figure S4B).

Next, we performed 3D dSTORM imaging of the endogenous ASC specks. Consistent with the 2D dSTORM images, the specks had an overall spherical shape with filaments occasionally reaching out from a dense core (Figure 3D, Supplementary Figure S5, Supplementary Figure S6, Supplementary Movie S1 and Supplementary Movie S2). The two-dimensional eccentricity for the ASC specks was determined to be 1.19, verifying more or less round specks, with an average (i.e. median) radius of gyration of $321 \mathrm{~nm}$ (Figure 3E, F, and Material and Methods). For some ASC specks, we observed a ring-like appearance (Figure 3H - L and Supplementary Figure S4A + Supplementary Figure S6). Some of the structures appeared less extended along the z-axis resulting in an overall disk-like shape (Figure 3I + Supplementary Movie S3 + Supplementary Movie S4) while others had a spherical, hollow shape (Figure 3K + L, Supplementary Movie S5). Although some specks appeared ring-like, the signal was not entirely excluded from the center. Hence, we hypothesized that local density differences may be responsible for the ring-like signal and that a complex of primary and secondary antibody could be sterically excluded from the dense center of the structure.

To test this hypothesis, we performed 3D super-resolution imaging using an ASC nanobody (23) to stain endogenous specks. By that, the size of the complex used for labelling is reduced by a factor of 3 . Following nanobody labelling and ASTORM imaging, the specks appeared as amorphous structures with no obvious organization (Figure 3M $-\mathbf{P}$ and Supplementary Figure S7). The structure remained spherical (Figure 3E) while the overall size of the specks, determined from the radius of gyration, was smaller (Figure 3F) and the total number of localizations within the speck decreased compared to specks stained with primary antibody and secondary $F_{a b}$ fragment (Figure 3G). None of the 134 structures observed exhibited a hollow center. DNA-PAINT confirmed our observation of a smaller speck size and could also resolve short filamentous extensions at the edge of the structure (Figure $30+$ P, Supplementary Figure S7B, Supplementary Figure S8 and Supplementary 
Movie S6). The absence of ring-like structures after nanobody labelling is consistent with our hypothesis of the dense regions within the speck limiting the accessibility of the labeling probe. The smaller radius of gyration cannot be totally explained by the high labeling density in the center and is attributed to the comparably low binding-affinity of the nanobody (apparent binding constant: $159.5 \pm 1.5 \mathrm{nM}(23))$. The nanobody has a single binding site, compared to two for a normal primary antibody, potentially explaining the higher labeling efficiency of the outer, lower-density regions of the speck by the antibody. 

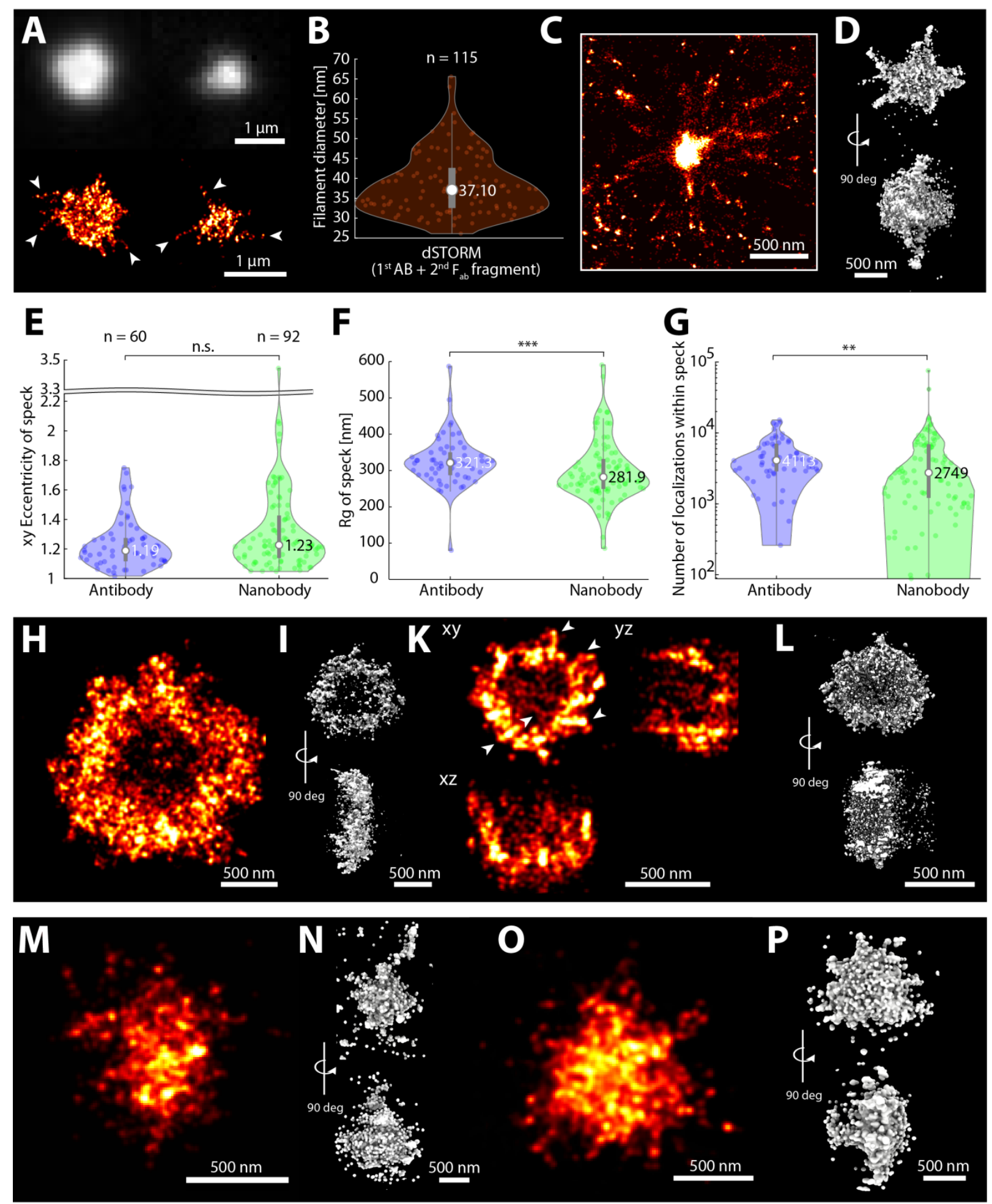

Figure 3 - Super-resolution imaging of the endogenous ASC speck. A) Two ASC specks stained with primary antibody and secondary $\mathrm{F}_{\mathrm{ab}}$ fragment in THP-1 cells exhibiting different sizes are shown after diffraction-limited widefield imaging (upper images) together with their 2D dSTORM reconstructions (lower images). B) The distribution of ASC filament diameters as measured in 2D dSTORM reconstructions. Circle in the violin indicates the median of the distribution. C) An ASC speck in BlaER1 cells imaged using DNA-PAINT super-resolution microscopy. Filaments are clearly observed reaching out from a dense core. D) A 3D dSTORM reconstruction of a single ASC speck stained with primary antibody and secondary $\mathrm{F}_{\mathrm{ab}}$ fragment in THP-1 cells. E-G) Comparison of the endogenous ASC speck parameters determined using primary antibody and secondary $\mathrm{F}_{\mathrm{ab}}$ fragment labeling (blue) and nanobody labeling (green). From the 2D dSTORM images, violin plots of the eccentricity (E), the radius of gyration (F) and the number of localizations (G) were determined. White circles in the violins indicate the median of the distribution. Statistical significance was assessed by Two-Sample Kolmogorov-Smirnov Test $(*) \mathrm{p}<0.05,(* *) \mathrm{p}<0.01,(* * *) \mathrm{p}<0.001$. H-L) A characteristic $2 \mathrm{D}$ reconstruction $(\mathrm{H})$ and a 3D 
reconstruction of an ASC speck (I) as well as a a z-stack projection of a 3D dSTORM reconstruction (K) and a 3D reconstruction of another ASC speck (L). Both exhibit a ring-like structure. In panel K, the arrows highlight filament-like structures. M-P) ASC specks stained with anti-ASC nanobodies. 2D (M) and 3D (N) reconstructions of two individual ASC specks imaged by dSTORM. O-P) 2D (O) and 3D (P) representations of an ASC speck imaged using DNA-PAINT. Short filamentous structures are resolvable at the edge of the dense speck core.

$\underline{\text { Two-color super-resolution imaging confirms accessibility differences within endogenous ASC specks }}$

To verify that the differences in morphology of the ASC specks observed from the two labeling approaches is not an artifact due to the details of the measurements, we performed two-color superresolution microscopy using both nanobody and antibody labeling on the same cells. Diffractionlimited widefield imaging showed that both labeling approaches specifically stained the ASC speck (Figure 4A). Consistent with our previous observation, the signal resulting from nanobody staining was smaller than the one obtained after antibody staining. Dual-color dSTORM reconstructions revealed the antibody-labeled specks have a diameter of about one micrometer in widefield and an $R_{g}$ of $321 \mathrm{~nm}$ (compared to $324 \mathrm{~nm}$ under single-labeling conditions, Table 1) with the nanobody staining localized in the center of the speck (Figure 4B and Supplementary Figure S9). Antibody labeling could also resolve filaments while the nanobody-stained structure mostly appeared amorphous and spherical. As discussed above, a subset of the ASC specks appeared hollow after staining with the antibody complex (Figure 4C). Strikingly, in these particles, the nanobody staining was restricted to the center of those specks with a significantly small $R_{g}(134 \mathrm{~nm}$, compared to $282 \mathrm{~nm}$ under single labeling conditions, Table 1). This is consistent with the higher affinity of the antibody outcompeting the nanobody in the lower density regions of the speck. Aligning the signals of both staining approaches along the center of mass of the antibody signal and merging the resulting structure confirmed the observation that the nanobody stained the center while the antibody complex was found more towards the speck periphery (Figure 4D). Hence, different labeling approaches bring out different features of the ASC speck. While it is typically beneficial to use small labels (55) (56), other factors such as binding affinity and structural density can also play a role. Hence, to confidently assess super-resolution structures, we recommend verifying the structure using different labeling approaches. 

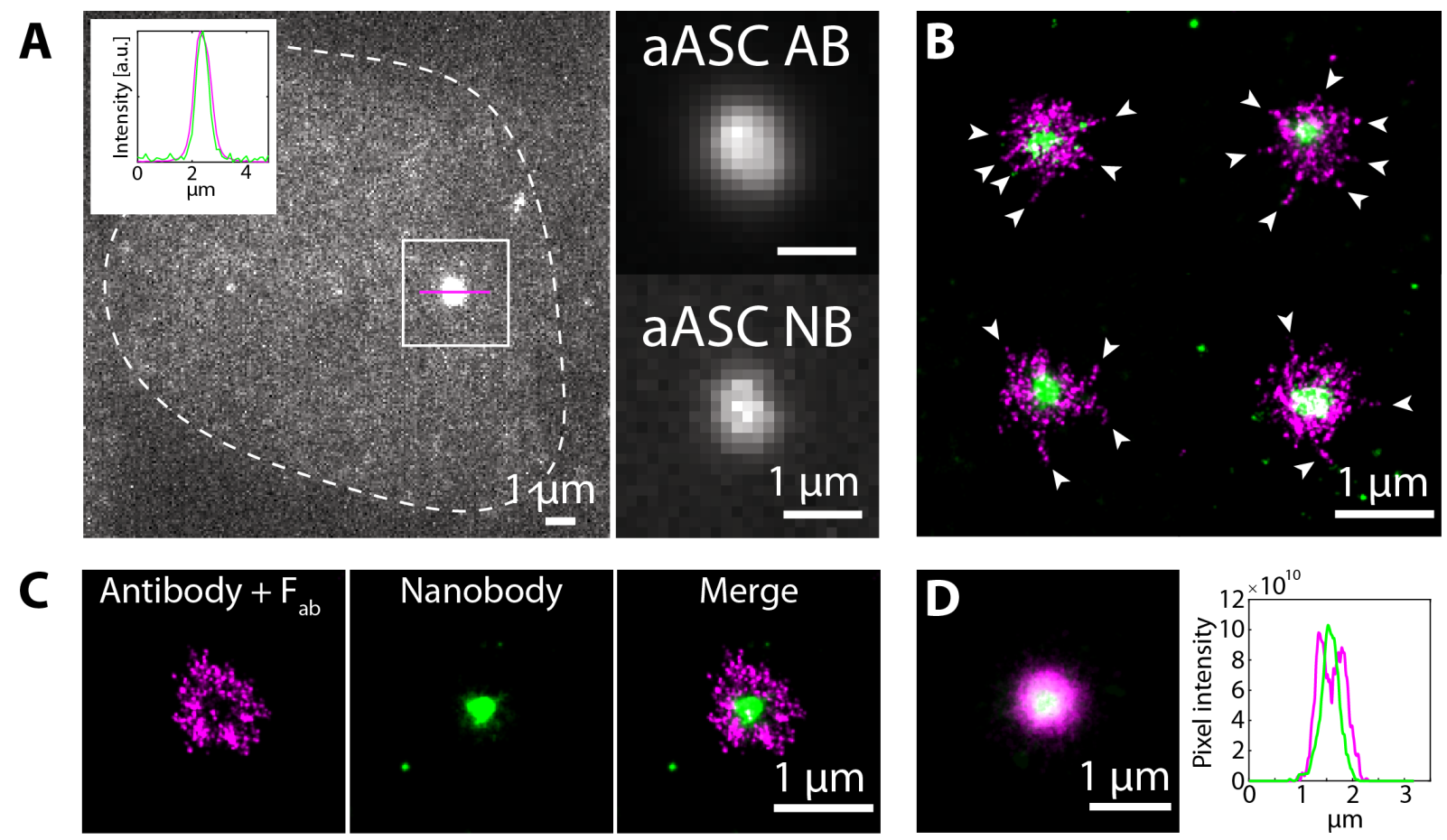

Figure 4 - Dual-color dSTORM of ASC specks simultaneously stained with primary antibodies/secondary $F_{a b}$ fragments and nanobodies. A) Diffraction-limited widefield imaging of an ASC speck stained with primary antibody and Alexa Fluor 647-labeled secondary $\mathrm{F}_{\mathrm{ab}}$ fragment (the signal of which is shown here) and DyLight 755-labeled nanobody. The dashed line shows the cell outline. The intensity profile of the ASC speck normalized to the peak of the intensity distribution along a cross-section of the speck (magenta line) is shown in the top left of the image (antibody signal: magenta; nanobody signal: green) showing that both staining approaches stain the same structure. The right part of the panel shows the boxed area split into the signal after antibody (top) and nanobody (bottom) staining. B) Four representative dual-color dSTORM reconstructions of ASC specks stained simultaneously with both antibodies and nanobodies (magenta: Alexa Fluor 647; green: DyLight 755). Arrows point towards filaments reaching out from the dense speck core. C) Dual-color dSTORM reconstruction of a speck showing a ring-like appearance after antibody $+\mathrm{F}_{\mathrm{ab}}$-staining (left), staining of the dense core by the nanobody (center) and the merge of both labeling strategies (right). D) Alignment of dual-color-labeled specks $(\mathrm{n}=35)$ along the center of mass of the antibody $+\mathrm{F}_{\mathrm{ab}}$ fragment staining (left) and the intensity profile along a cross-section through the aligned structure (right).

\section{$\underline{\text { ASC forms a scaffold of constant size that becomes denser with time }}$}

From the wealth of information that we gathered using widefield and super-resolution microscopy, we proceeded to quantitatively analyze the data. As the cytosolic labeling of ASC with nanobodies was less robust, we limited our analysis to the data collected using primary antibody and secondary $F_{a b}$ fragment labeling. We first manually segmented the cell and the speck such that we could calculate various parameters dependent on the characteristics of the cells and speck. Widefield images were collected before super-resolution microscopy was performed such that we had both widefield intensity and localization information available from the same cells. By plotting the total widefield intensity as a function of cell area, a clear correlation is observed (Supplementary Figure S10A) showing that larger cells express more ASC protein. This is also true if we plot the total number of localizations versus cell size (Supplementary Figure S10B). In fact, the total number of localizations measured using dSTORM correlates well with the total widefield intensity, as one would expect (Supplementary Figure S10C + D). If we normalize the total intensity by the cell area, the normalized intensity no longer depends on cell size (Supplementary Figure S10E). This suggests that the concentration of ASC is constant across different cells. 
Next, we investigated how the size of the speck varies with cell properties. The size of the speck, determined either by manual segmentation of the speck or via the calculation of the 2D radius of gyration, was found to increase with cell size (Supplementary Figure S10F, Figure 5A). To investigate how the radius of gyration depends on other parameters, we normalized out the cell-area dependence (Supplementary Figure S10K see Materials and Methods). Interestingly, the radius of gyration only weakly depends on the amount of ASC within the speck (Supplementary Figure S10L, M). The same trend was found when we quantified the speck size manually via the occupied area (Supplementary Figure S10G - I). As speck assembly is a dynamic process, we investigated the progression of the assembly process. Although we measured fixed samples, we took advantage of the fact that recruitment of ASC to the perinuclear speck upon NLRP3 inflammasome activation occurs stochastically in the different cells we measured (43) (16). We used the ratio of ASC signal in the speck with respect to the total amount of ASC within the entire cell as a metric for the progression of speck assembly, i.e. pseudo time. To calculate this ratio, we utilized the number of localizations as this is more reliable. As a verification that our pseudo time classification functions correctly, we observed that the widefield intensity of the speck, as well as the number of localizations within the speck increased with ASC recruitment (Supplementary Figure S10N - O). In addition, the cytosolic, nonspeck ASC signal consistently decreased with the pseudo time regardless of whether we quantified it using the number of localizations, the cluster density or the localization density (Supplementary Figure S10R - T). Due to the low level of signal remaining in the cytosol upon ASC recruitment to the speck, the autofluorescence signal becomes a significant fraction of the entire signal and a clear decrease in the integrated widefield intensity with the pseudo time is not observed (Supplementary Figure S10Q). Hence, recruitment of ASC determined via the number of localizations was used to determine a quasi-chronological ranking (pseudo time) of the complexes. A plot of the radius of gyration versus pseudo time showed little change in the size of the speck with pseudo time (Supplementary Figure 10U). This also holds true for the speck area (Supplementary Figure 10V). As speck size scales with cell area, the spread in $R_{g}$ with cell area could potentially hide the trend. Hence, we used the normalized speck size to see if we observe an increase of speck size with pseudo time. The normalized radius of gyration of the speck (corrected for the correlation with cell area) only increases slightly during the course of ASC recruitment (Figure 5B). Similarly, when we normalized out the increase in speck area with cell size, only a small increase in $\mathrm{R}_{\mathrm{g}}$ with pseudo time is observed (Supplementary Figure S10P). We also plotted cell area with pseudo time (Supplementary Figure S10W). Here, no correlation was observed. Although this was expected, it also verifies that the endogenous ASC speck formation is complete before pyroptosis and cell shrinkage is triggered. If the radius of gyration does not depend on pseudo time, but the ASC content does, then we would expect the ASC density in the speck to increase with time. We investigated the speck density (localizations per area) and found it to be largely independent of the cell area (Supplementary Figure S10X) but clearly increasing with ASC recruitment (Figure 5C). In line with this observation, there is a clear correlation between the speck density and the amount of ASC within the speck, calculated either via the integrated widefield intensity within the speck (Supplementary Figure S10Y) or the number of localizations within the speck (Supplementary Figure S10Z). Hence, we conclude that the density rather than the size of the speck increases with ASC recruitment.

To visualize potential structural rearrangements of the speck, we plotted the endogenous ASC specks as a function of pseudo time (Figure 5D). No clear structural progression was visible. To look for more subtle changes during ASC recruitment, we divided specks into three groups according to the fraction of recruited ASC. We aligned the complexes in each group to their center of mass. The resulting sum projection showed an increased tendency to form a ring-like structure at later stages of recruitment, which is consistent with the observation of the specks become denser with time and thereby exclude the antibody from the center of the speck (Figure 5C). 
A

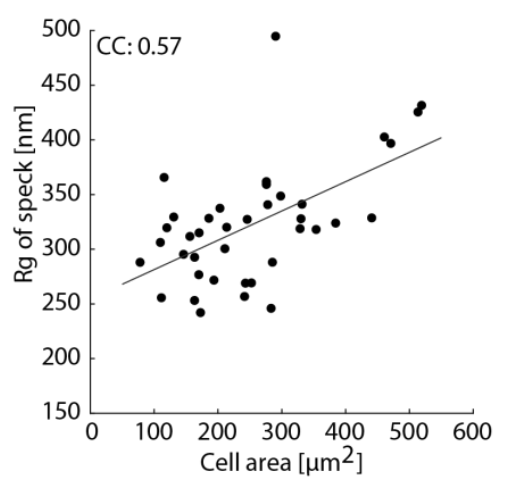

D
B

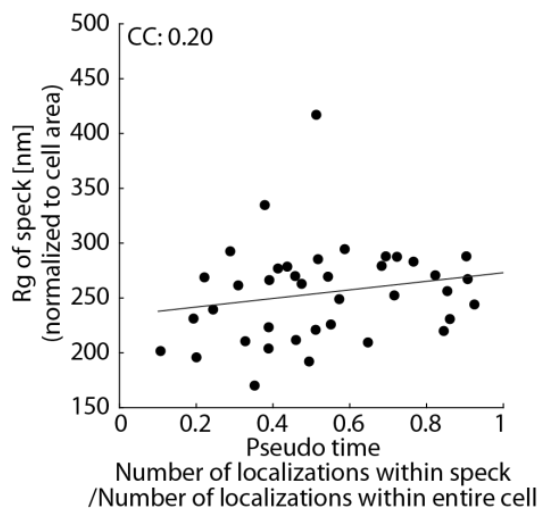

C

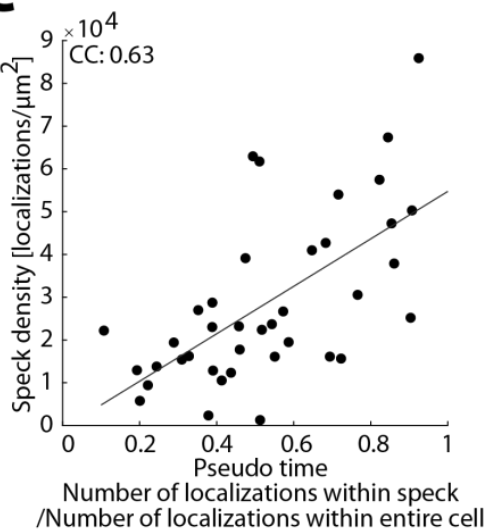

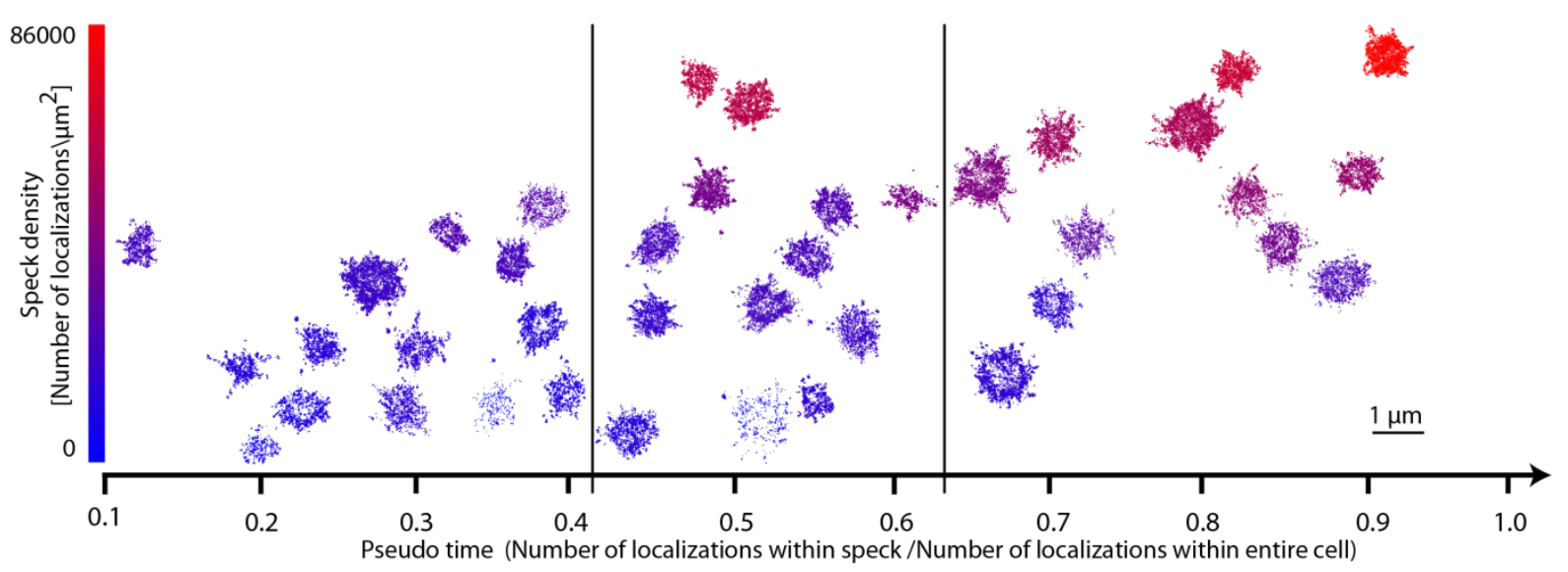
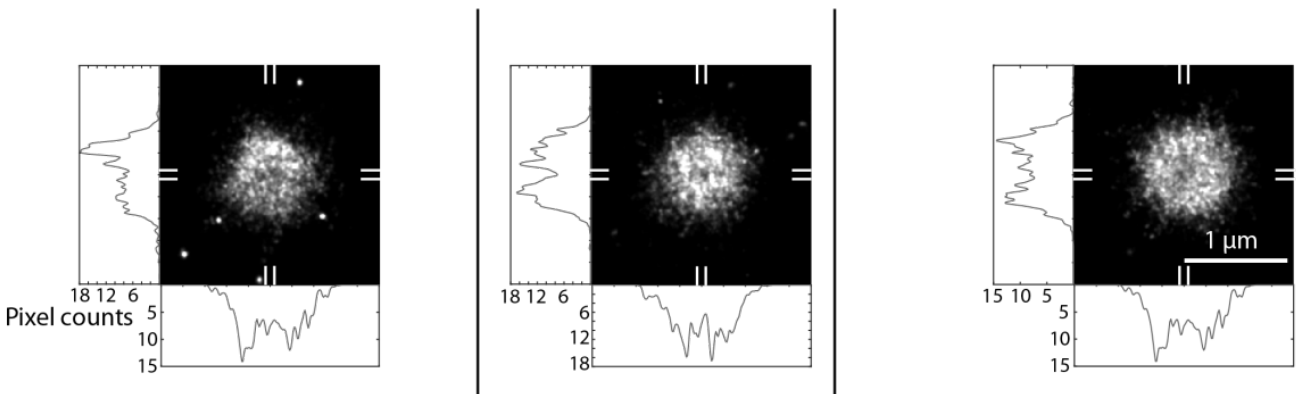

Figure 5 - Dynamics of endogenous ASC speck formation. A) - C) Scatter plots of speck size (measured as the radius of gyration) as a function of the cell area (A), the speck size, normalized to cell area versus the fraction of the total localizations located within the speck (pseudo time) (B) and the speck density (measured as number of localizations per $\mu \mathrm{m}^{2}$ ) as a function of pseudo time (C). Larger cells form larger specks and the normalized speck size stays almost constant with increasing pseudo time whereas the speck density increases with pseudo time. CC: Pearson correlation coefficient. D) Plot of individual speck structures as a function of the pseudo time. The speck density is color-coded (scale bar is shown on the left) and specks are positioned in the vertical direction approximately according to their density. Specks were separated into three time bins (separated by the vertical black lines) and the super-resolution structures aligned and summed (lower panels). Horizontal and vertical cross-sections are shown (determined by averaging along the marked 9 pixel wide regions indicated on the periphery with white lines). The averaged speck structure appears more ring-like at late time points compared to early time points.

Taken together, our data suggest that the speck forms initially as a loose scaffold of intertwined filaments whose size depends on the cell area and which becomes denser over time by recruiting and incorporating more ASC (schematically shown in Figure 6). 
A

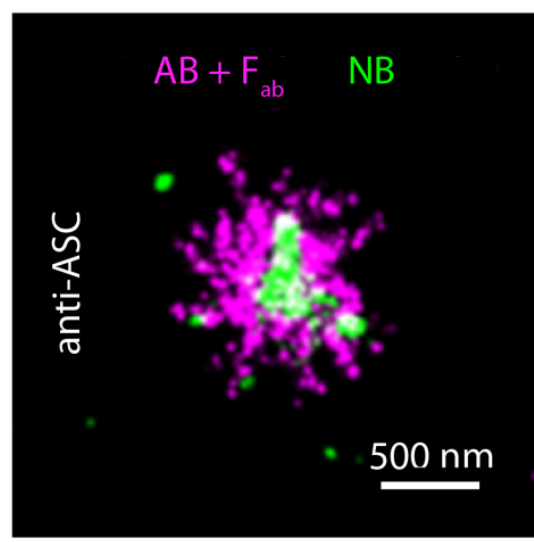

C

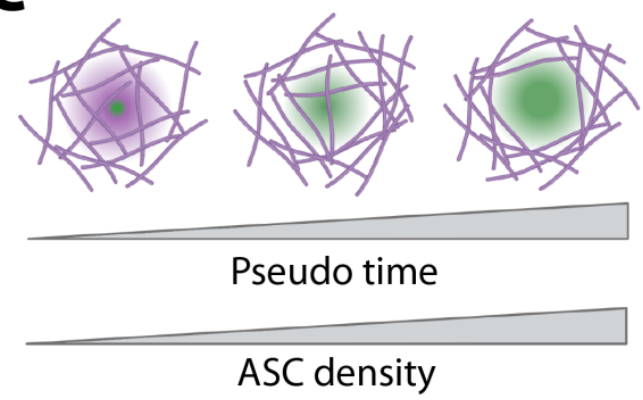

B
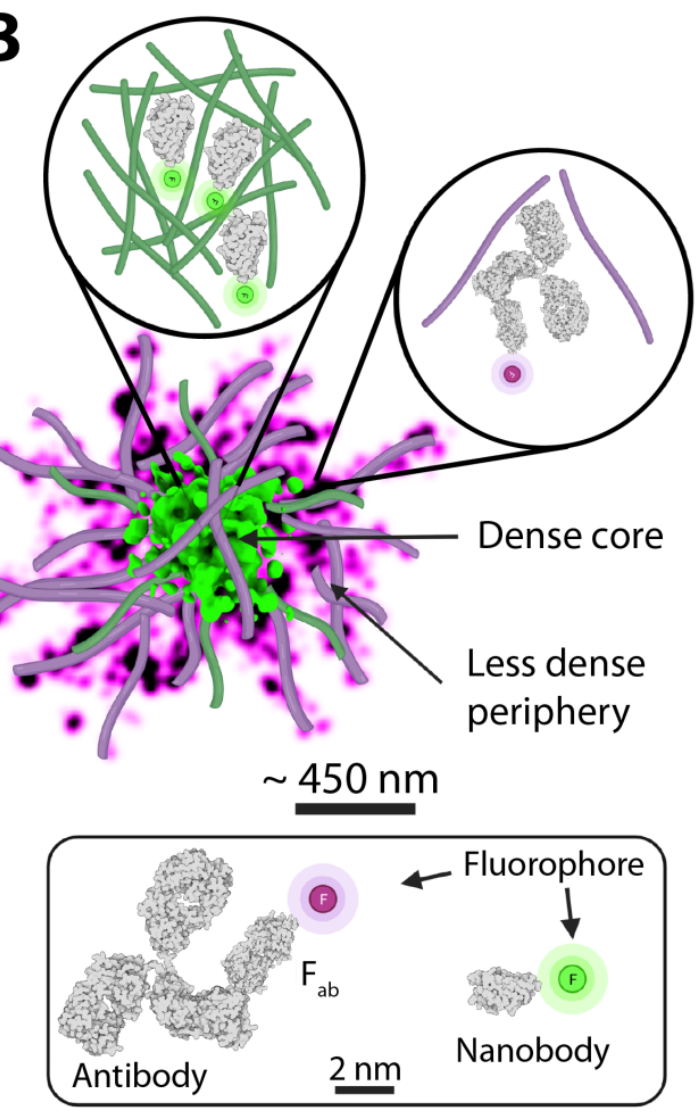

Figure 6 - Model of endogenous ASC speck formation. A) 2-color dSTORM reconstruction of an ASC speck stained with primary anti-ASC antibody (AB) and secondary Alexa Fluor 647-labeled $\mathrm{F}_{\mathrm{ab}}$ fragment and a DyLight755-labeled anti-ASC nanobody (NB). Notably the nanobody signal is located in the center whereas the antibody $+F_{a b}$ staining is observed more towards the periphery of the structure The scale bar corresponds to $500 \mathrm{~nm}$. B) Model for the supramolecular structure of the ASC speck. Modeled filaments are overlayed with the Alexa Fluor 647 structure shown in panel A and a 3D reconstruction of an ASC speck labeled with the DyLight755-conjugated anti-ASC nanobody. The structure is characterized by a dense core and a less dense periphery. Inserts on the top illustrate that the antibody- $\mathrm{F}_{\mathrm{ab}}$ fragment complex is for a subset of the specks excluded from the dense core due to its larger size whereas the smaller nanobody can label the central proportion of the structure. The insert at the bottom of panel B illustrates the size difference between the labeling probes. C) Illustration of speck formation over time. The ASC speck forms from intertwined filaments assembling into a scaffold whose size depends on the cell size. Early stages of speck formation are characterized by a loose assembly into which the antibody and nanobody can penetrate. Further ASC recruitment into the speck leads to denser structures but only marginally increases its size. Antibody $+\mathrm{F}_{\mathrm{ab}}$ fragment staining is sterically excluded from the dense center of the structure resulting in an overall ring-like appearance. The smaller nanobody stains the dense core of the speck but is washed away from the less dense regions of the structure due to its lower affinity.

\section{Discussion}

The supramolecular NLRP3 inflammasome complex is a central component of the innate immune system driving the maturation of the proinflammatory cytokines IL-1 $\beta$ and IL-18 as well as pyroptosis, a proinflammatory form of cell death. The protein ASC is critical during inflammasome formation and, upon cell stimulation, is recruited into a single, condensed structure, the ASC speck. How the ASC speck forms, the occurrence of possible intermediates and the organization of the final structure remains difficult to study due to the small size and heterogeneity of the complex. Here, we applied dSTORM and DNA-PAINT, super-resolution techniques, to investigate the organization of endogenous ASC in the speck as well as in the cytoplasm. dSTORM measurements were performed on a total of 358 cells and 251 specks were analyzed in detail. Treatment of the cells with LPS and 
nigericin, led to robust induction of ASC specks with a broad distribution in ASC content (Figure 1). In diffraction limited wide-field microscopy, the complexes appear as spherical structures with little morphological features observable. Hence, our motivation to use super-resolution microscopy to study the organization of ASC in the speck as well as in the cytoplasm.

\section{ASC associates into a spherical speck in activated cells}

We performed a cluster analysis on the non-speck bound cytosolic ASC fraction, which revealed that the oligomeric size of the vast majority of ASC signals remained unchanged between unstimulated and speck-containing cells (Figure 2E $+\mathbf{F}$ ). Considering the resolution of our images and the size of the labels, we measured an average $R_{g}$ in unstimulated cells of $\sim 15 \mathrm{~nm}$ (Figure 2F). This is similar in size to an atomic force microscopy (AFM) study that measured the dimensions of full-length human ASC assemblies, which organized into disc-like oligomers of $1 \mathrm{~nm}$ in height and $\sim 12 \mathrm{~nm}$ in diameter in vitro (57). Upon stimulation, we observe a small increase in the number of larger clusters in the cytosol. This could be due to preassembly of a portion of ASC into higher-order ASC oligomers that later associate into the speck as has been suggested in the literature $(7,58)$. Western blot analysis of inflammasome activated cells found different levels of ASC multimerization in addition to the ASC speck with a large proportion of the protein being dimeric (31).

In our systematic measurements of ASC speck formation, the cells either showed a single speck or the ASC protein appeared homogenously distributed throughout the cell. A clear decrease or enhancement of ASC as a function of distance from the speck is not see in our data (e.g. Figure 2C), consistent with the observations of Cheng et al. (43). This is not surprising since the entire pool of ASC is recruited into the ASC speck within a few minutes during complex formation (16) (7) (43) (59). We were still able to detect differences in the cytosolic content of ASC in speck containing cells. This could indicate that, for the endogenous protein, speck formation is fast in the beginning and then slows down. The lowest percentage of ASC recruitment we observed was 10\% suggesting that ASC recruitment, up until this point, occurs too quickly to be stopped by fixation. Our observation that it is mostly the speck density but not the speck size that increases with the percentage of ASC being recruited into the speck seemingly contradicts the observations made by Cheng et al, where an increase in speck size was observed in live cells with time (43). However, the increase in speck size occurs over a time interval of ca $100 \mathrm{~s}$, which would be too fast to trap with our fixation methods.

Interestingly, the specks we recorded were smaller in size than those measured previously upon overexpression of ASC $(20,39)$. As we observed an increase in the radius of gyration with total ASC content, this is not surprising (Figure 5A). However, NLRP3 inflammasome formation has recently been shown to occur at the MTOC (60) and a correlation has been observed between the size of the MTOC and the cell size (at least in C. elegans) (61). This could provide an alternative explanation for the size dependence of the ASC speck and may indicate that the MTOC provides a scaffold for assembly of the speck.

To reliably quantify the attributes of the amorphous, heterogeneous speck, we combined the results from 251 specks. The endogenous ASC speck has a size variation, determined from the 2D radius of gyration, ranging from 250 to $500 \mathrm{~nm}$ with an average radius of gyration of $321 \mathrm{~nm}$ (Figure 3F). This agrees with previously published values for the endogenous structure (31). Typically, one would expect the size of the speck to increase during assembly and hence the distribution of sizes depends, in part, on the stage of assembly. However, when calculating the amount of ASC located within the speck relative to the total amount of ASC within the cell (i.e. our pseudo time), we found that the speck size depends only to a minor degree on the amount of recruited ASC (Figure 5B).

\section{The ASC speck is formed of a dense core with protruding filaments}

We found the majority of specks appeared as amorphous objects with an overall spherical structure, as determined from the calculated eccentricity. These results are consistent with previous observations ( 7 , 
25). In addition, we observed that some specks exhibit filamentous extensions protruding from the dense core. Although several studies suggested that the ASC speck is made up of intertwined filaments (20) (18) (24) (23), this has not yet been confirmed for the endogenous, unperturbed structure inside cells. We observe a dense core with filaments protruding from the endogenous ASC speck, which would be consistent with this hypothesis.

We observed filaments of varying length and thickness. Some specks exhibit many short and faint fibrils (Figure 3 M-P) while others show a small number of longer filaments protruding from the edge of the speck center (Figure 3C). The thin fibrils are reminiscent of the fibrils resolved by EM at the edge of the in vitro formed ASC assembly (20). A similar variation in thickness of the filaments protruding from the dense speck core has been observed in immortalized $\mathrm{ASC}^{-/-}$bone marrow-derived macrophages (BMDMs) (18). Interestingly, electron microscopy data of in vitro assembled structure from full-length human ASC protein suggested that individual ASC filaments can laterally stack via the exposed CARD domains and the authors raise the question of whether this could also happen in the endogenous structure (15). The average filament thickness we obtained on the above mentioned filaments agrees with the thickness reported for a single filament $(\sim 16 \mathrm{~nm})(15)$ suggesting that, for the majority of filaments, lateral stacking does not take place.

It is important to mention that the majority of our experiments were conducted in caspase-1 knock-out cells. Hence, we cannot exclude that the lack of caspase-1 binding to ASC has an influence on the appearance of the speck. Experiments performed using DNA-PAINT on BlaER1 cells that express caspase-1 showed the same dense, amorphous core with protruding filaments. This strongly suggests that the measured structures are indicative of the morphology of the ASC speck and is consistent with what has been observed in experiments in cells with ASC overexpression (20) (39) (18). However, in comparison to the previous observations of the speck formed after overexpression (20) (39), we found fewer and shorter filaments protruding from the endogenous ASC speck core with the majority of filaments being $500 \mathrm{~nm}$ long or shorter. It is conceivable that overexpression leads a larger number of filaments emanating from the formed speck. Alternatively, caspase-1 may also influence the filament length as our measurements in BlaER1 cells that express caspase-1 showed longer filaments than the ones observed in THP-1 caspase-1 knock-out cells (Figure 3C + Supplementary Figure S4B). The longer filament lengths are in line with a previous report where in vitro experiments measured filament lengths of 500-2000 nm for full length mouse ASC (14). However, we cannot entirely rule out the differences in filament length are due to labeling artifacts in one or the other super-resolution approaches.

An overall ring-like appearance of the ASC speck has been proposed since its discovery (3). However, the question of how the ring-like assembly and the irregular structure made up of intertwined filaments relate to each other remains unanswered (35) (38) (36) (37). Here, we provide new data that offers an explanation for the observed ring-like structure. Complementing previous studies, which showed a ring-like assembly of ASC following antibody labelling, we used labels of different sizes, primary antibodies labeled with secondary $\mathrm{F}_{\mathrm{ab}}$ fragments and nanobodies. While primary antibody plus second $\mathrm{F}_{\mathrm{ab}}$ fragment labeling occasionally showed ring-like structures, simultaneous labeling with nanobodies revealed a dense core in the speck (Figure 4). Hence, we conclude that the endogenous speck can be divided into two different regions: a dense core and a less dense periphery (Figure 6). This would imply that the ring shape originates from the primary and/or secondary antibody being less likely to penetrate into the dense center of the structure and thus leading do a decrease in labeling efficiency at the center of the structure. Conversely, the less dense structures at the periphery are less efficiently labeled by the nanobody as it has only one binding site per protein with a relatively low $(\sim 160 \mathrm{nM})$ binding affinity. This demonstrates the importance of verifying various super-resolution structures using different labeling strategies. 


\section{Conclusion}

Taken together, we investigated the nanoscale organization of the endogenous ASC speck using superresolution microscopy. We found that the speck size was heterogenous and correlated with cell size. The speck contained a dense core with filaments protruding from the center of the speck. This was observed for dSTORM and DNA-PAINT measurements in THP-1 cells as well as DNA-PAINT measurements in BlaER1 cells. These results are consistent with the model of the ASC speck being an assembly of intertwined filaments. By using differently sized labels, we found that the ring-like appearance of the speck is a result of the labeling approach and limited access of the primary antibody and/or $F_{a b}$ fragment to the dense core of the speck (Figure 4). Conversely, the small nanobody was able to label the center of the speck but was less efficient in labeling the low density periphery regions due to the lower affinity of this nanobody. Hence, it is important to verify super-resolution structures using different labeling approaches. Finally, by measuring the fraction of recruited ASC, we hypothesize that speck formation starts with a loose scaffold that becomes denser but only marginally larger during ASC speck formation.

\section{Materials and Methods}

If not stated differently all reagents where purchased from Thermo Fisher Scientific, Massachusetts, USA.

Cultivation of cells and activation of the NLRP3 inflammasome

For the experiments performed with THP-1 caspase- 1 knock-out cells, the THP-1 cells were cultivated at $37^{\circ} \mathrm{C}, 5 \% \mathrm{CO}_{2}$ in Roswell Park Memorial Institute 1640 medium (21875034) supplemented with $10 \%(\mathrm{v} / \mathrm{v})$ heat-inactivated fetal bovine serum (FBS) (10500064), $1 \mathrm{mM}$ sodium pyruvate (11360039) and $100 \mathrm{U} / \mathrm{ml}$ Penicillin/Streptomycin (15140122), and maintained at a density between $1 \times 10^{5}$ and $1 \times 10^{6}$ cells per milliliter. One day prior to seeding the cells, coverslips (1.5, Menzel Gläser, $18 \mathrm{~mm}$ ) were coated with $0.01 \%$ poly-1-ornithine solution (A-004-C, Merck-Millipore, Massachusetts, USA) in the dark. Cells were seeded at a density of $\sim 75 \times 10^{3} / \mathrm{cm}^{2}$ in culture medium supplemented with 100 ng/ml Phorbol 12-myristate 13-acetate (PMA) (BML-PE160-0001, Enzo Life Sciences, Lörrach, Germany) and differentiated into macrophage-like cells for three days. To increase NLRP3 protein expression, cells were primed for three hours with $1 \mu \mathrm{g} / \mathrm{ml}$ Lipopolysaccharide (LPS) from E. coli K12 cells (Ultrapure tlrl-peklps, Invivogen, San Diego, California, USA). To suppress cell death due to caspase activity, cells were subsequently treated with $20 \mu \mathrm{M}$ of the pan-caspase inhibitor Z-VAD (tlrl-vad, Invivogen) for 60 mins. The NLRP3 inflammasome response was activated by incubating the cells with $10 \mu \mathrm{M}$ Nigericin (N7143-5MG, Sigma Aldrich, Missouri, USA) for 90 mins.

ASC immunofluorescence with primary antibody and secondary $\mathrm{F}_{\mathrm{ab}}$ fragment and for double-labeling with an ASC nanobody

All steps were performed at room temperature if not stated differently. Cells were washed once with phosphate-buffered saline (PBS) solution to remove remaining serum proteins, fixed for 15 mins in the dark with 4\% paraformaldehyde (PFA) (E15710-S, Electron Microscopy Sciences, Pennsylvania, USA) in PBS. Subsequently, PFA was rinsed once with PBS and then quenched by rinsing once with $0.1 \mathrm{M} \mathrm{NH}_{4} \mathrm{Cl}$ (254134, Sigma-Aldrich) in PBS followed by a $15 \mathrm{~min}$ incubation with $0.1 \mathrm{M} \mathrm{NH}_{4} \mathrm{Cl}$ in PBS. Permeabilization and blocking was done for 30 mins in 10\% normal goat serum (NGS) (16201), 0.5\% Triton X-100 (T8787, Sigma-Aldrich) in PBS followed by a washing step with PBS and 30 mins 
incubation in Image-iTFX Signal enhancer (I36933). After two additional PBS washing steps, cells were incubated overnight at $4^{\circ} \mathrm{C}$ with purified monoclonal mouse anti-human ASC antibodies (TMS1) (clone HASC-71) (Biolegend, California, USA) at a final concentration of $10 \mu \mathrm{g} / \mathrm{ml}$ diluted in $10 \%$ NGS $+0.5 \%$ Triton X-100 in PBS. $25 \mu 1$ of this solution was applied to the fixed cells on a coverslip. The plasmid encoding for the ASC nanobody was provided by Prof. Dario Alessi, University of Dundee via the MRC - Protein Phosphorylation and Ubiquitylation Unit [DU 54832]. The nanobody encoding sequence with a C-terminal cysteine was amplified for labeling and expressed at the protein production core facility of the Max-Planck-Institute for Biochemistry in Martinsried, Germany. Parts of the nanobody were labeled with Alexa Fluor 647 in house as described in (62) and at Nanotag Biotechnologies GmbH, Göttingen, Germany. In the case of dual-color labeling, DyLight755-labeled anti-ASC nanobody was purchased from Nanotag and mixed into the antibody solution at $1 \mu \mathrm{g} / \mathrm{ml}$ final concentration. Non-specific sticking of antibody and nanobody was minimized by washing three times with $0.1 \%$ Triton X-100 in PBS. Labeling with secondary F(ab')2-goat anti-mouse $\operatorname{IgG}(\mathrm{H}+\mathrm{L})$ cross-adsorbed Alexa Fluor 647-labeled $F_{a b}$ fragment (A-21237) was performed at $200 \mathrm{ng} / \mathrm{ml}$ final concentration in $10 \% \mathrm{NGS}+0.5 \%$ Triton X-100 in PBS for $1 \mathrm{hr}$ followed by three washing steps with $0.1 \%$ Triton X-100 in PBS and postfixation with 3\% PFA in PBS for 10 mins. The specificity of the staining procedure was confirmed by staining THP-1 caspase- 1 knock-out cells only with the $\mathrm{F}_{\mathrm{ab}}$ fragment without previous administration of the primary antibody as well as by applying the staining protocol to THP-1 ASC knock-out cells (Supplementary Figure S10).

\section{$\underline{\text { ASC immunofluorescence with ASC nanobody }}$}

THP-1 Caspase-1 knock-out cells were activated for the NLRP3 inflammasome, fixed and quenched as described above followed by permeabilization with $0.05 \%$ Saponin (47036, Sigma-Aldrich), 1\% BSA (A7030, Sigma-Aldrich), and $0.05 \% \mathrm{NaN}_{3}$ (S2002, Sigma-Aldrich) in PBS for 20 mins. Afterwards, the sample was wash for 2 mins with PBS and then the sample was blocked for 30 mins with Image-iTFX Signal Enhancer (R37107). The sample was then washed twice for 2 mins with PBS and stained against ASC with $1 \mathrm{ug} / \mathrm{ml}$ Alexa Fluor 647-labeled nanobody and postfixed for $10 \mathrm{mins}$ in 3\% PFA in PBS. The specificity of the staining was confirmed using THP-1 ASC knock-out cells (Supplementary Figure S12).

\section{Confocal imaging}

For the confocal imaging shown in Figure 1, the samples were embedded in ProLong Gold (P10144) on standard glass slides for at least one day before imaging. Microscopy was then performed on a Leica SP8 STED 3x equipped with a 470-670nm white light laser and a $100 \mathrm{x}$ PlanApo /NA 1.4 objective. The spinning disk confocal microscopy images shown in Supplementary Figure $\mathbf{S 1 1}$ were measured on a Zeiss Cell Observer SD. Alexa Fluor 647 and DAPI were excited using a $639 \mathrm{~nm}$ and $405 \mathrm{~nm}$ laser, respectively. Fluorescence was separated using a $660 \mathrm{~nm}$ longpass filter and recorded on the two Evolve 512 electron-multiplying charge-coupled device cameras (Photometrics) of the system equipped with a 525/50 and a 690/50 bandpass filter, respectively.

\section{dSTORM imaging and analysis}

Samples were imaged on a flat field-optimized widefield setup as described before (48). Briefly, lasers of $405 \mathrm{~nm}, 642 \mathrm{~nm}$ and $750 \mathrm{~nm}$ were expanded and reflected into a 60 x objective (CFI60 PlanApo 
Lambda 60x / NA 1.4, Nikon) using a custom dichroic mirror (ZT405/561/642/750/850rpc, Chroma). Fluorescence emission was imaged onto a sCMOS camera (Prime, Photometrics) using one of two emission filters (ET700/75M and ET810/90m, Chroma) combined with a short-pass filter (FF01$842 / \mathrm{SP}$, Semrock) and a tube lens of $200 \mathrm{~mm}$ focal length. The microscope was controlled using Micromanager (63), (64). Widefield images were collected prior to dSTORM recordings. We typically recorded between $20-80 \mathrm{k}$ frames at $10 \mathrm{~ms}$ exposure time. For 3D dSTORM, we introduced a cylindrical lens ( $\mathrm{f}=1000 \mathrm{~mm}$, Thorlabs LJ1516RM-A) into the emission path. Single- and dual-color single-molecule localization microscopy (SMLM) imaging was carried out with an optimized SMLM buffer, as described previously (65). Single molecules were localized using an sCMOS-specific localizer routine introduced by Huang et al. (66) and included in a custom MatLab program used for data analysis. To exclude non-specific localizations, filter parameters were adjusted using datasets acquired using ASC knock-out THP-1 cells. Drift correction was performed using the Redundancy Cross-Correlation (RCC) algorithm introduced by Wang et al. (67). The cells and specks were manually segmented. Cytoplasmic localizations were clustered using DBSCAN where a cluster was defined as a group of $10-300$ localizations within a search radius of $70 \mathrm{~nm}$ (46) or Ripley's $\mathrm{K}$ function (47). Super-resolution images were reconstructed using ThunderStorm (68) with 10x magnification and applying a Gaussian blur of one pixel $(10.6 \mathrm{~nm})$. For dual-color data sets, the localizations in the DyLight 755 channel were registered to the ones in the Alexa Fluor 647 channel by an affine transformation calculated from widefield images of immobilized TetraSpeck Microspheres (T7279) on a plasma cleaned coverslip recorded in both channels. 3D representations were rendered using Chimera X (69).

The eccentricity, the radius of gyration and the number of localizations of the specks were calculated from the manually segmented localizations within the structure. The radius of gyration was calculated as the square root of the sum of the variances of the $\mathrm{x}$ and the $\mathrm{y}$ coordinates of the individual localizations with each speck. As, in most cases, the specks are amorphous, spherical structures and we have significantly better resolution in the radial dimension, we limited the calculated radius of gyration to two-dimensions. The eccentricity of the individual specks was determined from the localizations by first calculating the covariance matrix of the individual localizations. From the covariance matrix, the eigenvectors were calculated, which provide a measure for how elliptical the distribution of localizations is and calculates the direction of the major and minor axes of the ellipse. We then take the square root of eigenvectors, which give a measure of length for the different axes. Finally, the eccentricity is calculated by taking the ratio of the major axis (square root of the maximum eigenvector) to the minor axis (square root of the minimum eigenvector). Circular objects have an eccentricity near 1.

For the quantitative image analysis, measurements of the integrated widefield intensity were corrected by subtracting background counts determined individually for each field of view by averaging pixel counts in an area absent of cellular signal. To calibrate for the experimental differences observed between measurement days, the widefield intensity per cell area and the number of detected localizations per widefield intensity were corrected separately for each measurement day. For this, we used the correlation of fluorescence intensity with cell size and the number of localizations versus widefield intensity. The correlation was determined for each day by fitting the data with a line and the slope was scaled to correspond to the maximum of all experiment days. A correction factor was determined for each measurement day and used to rescale the measurements to the measurement day with the largest slope. The scaled widefield intensity and number of localizations were then used for the ensuing analyses. Supplementary Figure S13 illustrates the applied correction procedure. 
The cell area dependence of the radius of gyration $\left(R_{g}\right)$ was normalized out by first plotting the $R_{g}$ against the cell area and fitting the data with a line. Subsequently for each data point, the product of the slope of the line and the value of the cell area was calculated and subtracted from the $R_{g}$ value. The speck area was normalized analogously.

\section{$\underline{\text { BlaER1 cell preparation and DNA-PAINT imaging }}$}

BlaER1 ASC-TagRFP cells were cultivated as described for the THP-1 cells. Cells were transdifferentiation for 7 days at 60,000 cells $/ \mathrm{cm}^{2}$ in medium containing $50 \mathrm{ng} / \mathrm{ml} \mathrm{IL-3} \mathrm{(200-03} \mathrm{B,}$ Peprotech, New Jersey, USA), $50 \mathrm{ng} / \mathrm{ml} \mathrm{M-CSF} \mathrm{(300-25} \mathrm{B,} \mathrm{Peprotech)} \mathrm{and} 500 \mathrm{nM} \beta$-Estradiol (E8875, Sigma-Aldrich) in cell culture-treated plastic bottom slides (80826, ibidi, Gräfelfing, Germany). Cells were then trypsinized, transferred to glass bottom slides coated with poly-1-ornithine as described for THP-1 cells in cultivation medium without growth factors at the same density and incubated overnight at $37^{\circ} \mathrm{C}, 5 \% \mathrm{CO}_{2}$. The next day, the NLRP3 inflammasome was activated by priming the cells for $14 \mathrm{hrs}$ with $200 \mathrm{ng} / \mathrm{ml}$ Lipopolysaccharide (LPS) from E. coli K12 cells in medium followed by caspase inhibition with $20 \mu \mathrm{M}$ Z-VAD-FMK incubation for 60 mins as described for THP-1 cells and 3 hrs incubation with $6.5 \mu \mathrm{M}$ Nigericin. Cells were fixed and stained as described for THP-1 cells with the exception that a secondary donkey anti-mouse antibody (715-005-151, Jackson ImmunoResearch) conjugated to a P3 docking strand (TTCTTCATTA) was used instead of the $F_{a b}$ fragment. Specks were identified by excitation of TagRFP with a $561 \mathrm{~nm}$ laser, followed by bleaching the signal and DNA-PAINT data were recorded using a P3-Cy3B imager strand (AATGAAGA-Cy3B) at $1 \mathrm{nM}$ concentration and HiLo illumination at $1.2 \mathrm{~kW} / \mathrm{cm}^{2}$ excitation with the same laser at $100 \mathrm{~ms}$ camera exposure time for 8000 frames.

THP-1 cells imaged by DNA-PAINT were first labeled with a low amount of Alexa Fluor 647-labeled anti-ASC nanobody to facilitate identification of the speck. Subsequently, the nanobody conjugated to a P3 docking strand was applied and the structure was imaged using the complementary imager strand labeled with Cy3B at $0.5 \mathrm{nM}$ concentration. Images were recorded for 20,000 - 30,000 frames with an exposure time of $100 \mathrm{~ms}$ and a laser power density of $\sim 200 \mathrm{~kW} / \mathrm{cm}^{2}$.

Super-resolution images were reconstructed using the Picasso [51] and ThunderStorm [64] softwares and rendered with a Gaussian blur of one pixel corresponding to $13 \mathrm{~nm}$.

\section{Authors contributions}

I. M. G. and D. C. L. designed experiments, I. M. G., C. S. and D. C. L. analyzed the data, I. M. G., G. A. and G. M. P. labeled the nanobody, I. M. G. and G. M. P. prepared immunofluorescence samples, C. S. and I. M. G. recorded dSTORM data, S. S. and I. M. G. recorded DNA-PAINT data, C. S. prepared THP-1 knock-out cells, T. S. E. and C. S. helped with establishing the immunofluorescence protocols, D. C. L., V. H., S. M., R. J. and I. M. G. acquired funding, I. M. G., C. S. and D. C. L. wrote the manuscript with input from all other authors. 


\section{Financial Support}

I. M. G. gratefully acknowledges the financial support of the Studienstiftung des deutschen Volkes for a $\mathrm{PhD}$ fellowship. D.C.L gratefully acknowledges the financial support of the Deutsche Forschungsgemeinschaft (DFG, German Research Foundation) - Project-ID 201269156 - SFB 1032 Project B03 and Ludwig-Maximilian University of Munich via the Center for NanoScience (CeNS) and the LMUinnovativ initiative BioImaging Network (BIN).

\section{Conflicts of Interest}

The authors declare no conflict of interest.

\section{Acknowledgements}

We thank Fionan O'Duill for advice on cell culture and inflammasome activation. We would also like to thank Dr. Steffen Frey and Dr. Hansjörg Götzke from NanoTag Biotechnologies GmbH, Göttingen, Germany as well as Dr. Sabine Suppmann and Leopold Ulrich from the Protein Production Core Facility at the Max-Planck-Institute for Biochemistry, Martinsried, Germany for expressing and labeling nanobodies used in this study, and for fruitful discussions.

\section{References}

1. Martinon F, Burns K, \& Tschopp J (2002) The inflammasome: a molecular platform triggering activation of inflammatory caspases and processing of prolL-beta. Molecular cell 10(2):417426.

2. Conway KE, et al. (2000) TMS1, a Novel Proapoptotic Caspase Recruitment Domain Protein, Is a Target of Methylation-induced Gene Silencing in Human Breast Cancers. Cancer Research 60(22):6236-6242.

3. Masumoto J, et al. (1999) ASC, a novel 22-kDa protein, aggregates during apoptosis of human promyelocytic leukemia HL-60 cells. The Journal of biological chemistry 274(48):3383533838.

4. Howard AD, et al. (1991) IL-1-converting enzyme requires aspartic acid residues for processing of the IL-1 beta precursor at two distinct sites and does not cleave 31-kDa IL-1 alpha. The Journal of Immunology 147(9):2964-2969.

5. Thornberry NA, et al. (1992) A novel heterodimeric cysteine protease is required for interleukin-1 beta processing in monocytes. Nature 356(6372):768-774.

6. Wang L, et al. (2002) PYPAF7, a novel PYRIN-containing Apaf1-like protein that regulates activation of NF-kappa B and caspase-1-dependent cytokine processing. The Journal of biological chemistry 277(33):29874-29880.

7. Fernandes-Alnemri T, et al. (2007) The pyroptosome: a supramolecular assembly of ASC dimers mediating inflammatory cell death via caspase-1 activation. Cell Death \& Differentiation 14(9):1590-1604.

8. Hofmann K, Bucher P, \& Tschopp J (1997) The CARD domain: a new apoptotic signalling motif. Trends in Biochemical Sciences 22(5):155-156. 
9. $\quad$ Liepinsh E, et al. (2003) The Death-domain Fold of the ASC PYRIN Domain, Presenting a Basis for PYRIN/PYRIN Recognition. Journal of Molecular Biology 332(5):1155-1163.

10. Sahillioglu Ali C, Sumbul F, Ozoren N, \& Haliloglu T (2014) Structural and Dynamics Aspects of ASC Speck Assembly. Structure 22(12):1722-1734.

11. Vajjhala PR, Mirams RE, \& Hill JM (2012) Multiple binding sites on the pyrin domain of ASC protein allow self-association and interaction with NLRP3 protein. The Journal of biological chemistry 287(50):41732-41743.

12. Vajjhala PR, et al. (2014) Identification of multifaceted binding modes for pyrin and ASC pyrin domains gives insights into pyrin inflammasome assembly. J Biol Chem 289(34):23504-23519.

13. Chou JJ, Matsuo H, Duan H, \& Wagner G (1998) Solution Structure of the RAIDD CARD and Model for CARD/CARD Interaction in Caspase-2 and Caspase-9 Recruitment. Cell 94(2):171180.

14. Sborgi L, et al. (2015) Structure and assembly of the mouse ASC inflammasome by combined NMR spectroscopy and cryo-electron microscopy. Proceedings of the National Academy of Sciences 112(43):13237-13242.

15. Nambayan RJT, Sandin SI, Quint DA, Satyadi DM, \& de Alba E (2019) The inflammasome adapter ASC assembles into filaments with integral participation of its two Death Domains, PYD and CARD. The Journal of biological chemistry 294(2):439-452.

16. Cai $X$, et al. (2014) Prion-like Polymerization Underlies Signal Transduction in Antiviral Immune Defense and Inflammasome Activation. Cell 156(6):1207-1222.

17. Lu A, et al. (2014) Unified Polymerization Mechanism for the Assembly of ASC-Dependent Inflammasomes. Cell 156(6):1193-1206.

18. Dick MS, Sborgi L, Rühl S, Hiller S, \& Broz P (2016) ASC filament formation serves as a signal amplification mechanism for inflammasomes. Nature communications 7(1):11929.

19. Li Y, et al. (2018) Cryo-EM structures of ASC and NLRC4 CARD filaments reveal a unified mechanism of nucleation and activation of caspase-1. Proceedings of the National Academy of Sciences 115(43):10845-10852.

20. Franklin BS, et al. (2014) The adaptor ASC has extracellular and 'prionoid' activities that propagate inflammation. Nature Immunology 15(8):727-737.

21. Richards N, et al. (2001) Interaction between pyrin and the apoptotic speck protein (ASC) modulates ASC-induced apoptosis. The Journal of biological chemistry 276(42):39320-39329.

22. Martin-S\{\\{a\}\}nchez Fat, et al. (2020) ASC oligomer favor caspase-1 CARD domain recruitment after intracellular potassium efflux. bioRxiv:2020.2001.2027.921239.

23. Schmidt Fl, et al. (2016) A single domain antibody fragment that recognizes the adaptor ASC defines the role of ASC domains in inflammasome assembly. J Exp Med 213(5):771-790.

24. Baroja-Mazo A, et al. (2014) The NLRP3 inflammasome is released as a particulate danger signal that amplifies the inflammatory response. Nature Immunology 15(8):738-748.

25. Schneider KS, et al. (2017) The Inflammasome Drives GSDMD-Independent Secondary Pyroptosis and IL-1 Release in the Absence of Caspase-1 Protease Activity. Cell Reports 21(13):3846-3859.

26. Bryan NB, Dorfleutner A, Rojanasakul Y, \& Stehlik C (2009) Activation of Inflammasomes Requires Intracellular Redistribution of the Apoptotic Speck-Like Protein Containing a Caspase Recruitment Domain. The Journal of Immunology 182(5):3173-3182.

27. Bryan NB, et al. (2010) Differential splicing of the apoptosis-associated speck like protein containing a caspase recruitment domain (ASC) regulates inflammasomes. Journal of Inflammation 7(1):23.

28. Khare S, et al. (2012) An NLRP7-Containing Inflammasome Mediates Recognition of Microbial Lipopeptides in Human Macrophages. Immunity 36(3):464-476.

29. Lage SL, Dominical VM, Wong C-S, \& Sereti I (2019) Evaluation of Canonical Inflammasome Activation in Human Monocytes by Imaging Flow Cytometry. Frontiers in Immunology 10(1284). 
30. Li J-y, et al. (2018) Characterization of an NLRP1 Inflammasome from Zebrafish Reveals a Unique Sequential Activation Mechanism Underlying Inflammatory Caspases in Ancient Vertebrates. The Journal of Immunology 201(7):1946-1966.

31. Man SM, et al. (2014) Inflammasome activation causes dual recruitment of NLRC4 and NLRP3 to the same macromolecular complex. Proceedings of the National Academy of Sciences 111(20):7403-7408.

32. Masumoto J, Taniguchi S, \& Sagara J (2001) Pyrin N-terminal homology domain- and caspase recruitment domain-dependent oligomerization of ASC. Biochem Biophys Res Commun 280(3):652-655.

33. McConnell BB \& Vertino PM (2000) Activation of a Caspase-9-mediated Apoptotic Pathway by Subcellular Redistribution of the Novel Caspase Recruitment Domain Protein TMS1. Cancer Research 60(22):6243-6247.

34. McConnell BB \& Vertino PM (2004) TMS1/ASC: the cancer connection. Apoptosis 9(1):5-18.

35. Monie T \& Boyle J (2015) Insights into assembly of the macromolecular inflammasome complex. Inflammasome 1.

36. Chu LH, Gangopadhyay A, Dorfleutner A, \& Stehlik C (2015) An updated view on the structure and function of PYRIN domains. Apoptosis 20(2):157-173.

37. de Alba E (2019) Structure, interactions and self-assembly of ASC-dependent inflammasomes. Archives of biochemistry and biophysics 670:15-31.

38. Elliott EI \& Sutterwala FS (2015) Initiation and perpetuation of NLRP3 inflammasome activation and assembly. Immunological reviews 265(1):35-52.

39. Hoss F, Rodriguez-Alcazar JF, \& Latz E (2017) Assembly and regulation of ASC specks. Cellular and molecular life sciences : CMLS 74(7):1211-1229.

40. Bauernfeind FG, et al. (2009) Cutting Edge: NF-kB Activating Pattern Recognition and Cytokine Receptors License NLRP3 Inflammasome Activation by Regulating NLRP3 Expression. The Journal of Immunology 183(2):787-791.

41. Mariathasan S, et al. (2006) Cryopyrin activates the inflammasome in response to toxins and ATP. Nature 440(7081):228-232.

42. Gammon K (2013) Inflammation: A complex problem. Nature 502(7473):S86-S87.

43. Cheng J, et al. (2010) Kinetic properties of ASC protein aggregation in epithelial cells. Journal of Cellular Physiology 222(3):738-747.

44. Heilemann $M$, et al. (2008) Subdiffraction-Resolution Fluorescence Imaging with Conventional Fluorescent Probes. Angewandte Chemie International Edition 47(33):61726176.

45. Sieben C, Banterle N, Douglass KM, Gönczy P, \& Manley S (2018) Multicolor single-particle reconstruction of protein complexes. Nature Methods 15(10):777-780.

46. Ester M, Kriegel H-P, Sander Jor, \& Xu X (1996) A density-based algorithm for discovering clusters in large spatial databases with noise. $K d d$, pp 226-231.

47. Ripley BD (2016) The second-order analysis of stationary point processes. Journal of Applied Probability 13(2):255-266.

48. Douglass KM, Sieben C, Archetti A, Lambert A, \& Manley S (2016) Super-resolution imaging of multiple cells by optimized flat-field epi-illumination. Nature Photonics 10(11):705-708.

49. Zwettler FU, et al. (2020) Molecular resolution imaging by post-labeling expansion singlemolecule localization microscopy (Ex-SMLM). Nature communications 11(1):3388.

50. Jungmann R, et al. (2010) Single-Molecule Kinetics and Super-Resolution Microscopy by Fluorescence Imaging of Transient Binding on DNA Origami. Nano Letters 10(11):4756-4761.

51. Schnitzbauer J, Strauss MT, Schlichthaerle T, Schueder F, \& Jungmann R (2017) Superresolution microscopy with DNA-PAINT. Nature Protocols 12(6):1198-1228.

52. Rapino F, et al. (2017) C/EBP $\alpha$ Induces Highly Efficient Macrophage Transdifferentiation of B Lymphoma and Leukemia Cell Lines and Impairs Their Tumorigenicity. Cell Rep 19(6):1281.

53. Gaidt MM, et al. (2016) Human Monocytes Engage an Alternative Inflammasome Pathway. Immunity 44(4):833-846. 
54. Gaidt MM, Rapino F, Graf T, \& Hornung V (2018) Modeling Primary Human Monocytes with the Trans-Differentiation Cell Line BLaER1. Methods in molecular biology (Clifton, N.J.) 1714:57-66.

55. Moore RP \& Legant WR (2018) Improving probes for super-resolution. Nature Methods 15(9):659-660.

56. Ganji M, Schlichthaerle T, Eklund AS, Strauss S, \& Jungmann R (2021) Quantitative Assessment of Labeling Probes for Super-Resolution Microscopy Using Designer DNA Nanostructures. ChemPhysChem 22(10):911-914.

57. de Alba E (2009) Structure and interdomain dynamics of apoptosis-associated speck-like protein containing a CARD (ASC). The Journal of biological chemistry 284(47):32932-32941.

58. Santiveri CM, Oroz J, \& de Alba E (2014) A ring-like model for ASC self-association via the CARD domain. Inflammasome 1:44-54.

59. Sagoo $\mathrm{P}$, et al. (2016) In vivo imaging of inflammasome activation reveals a subcapsular macrophage burst response that mobilizes innate and adaptive immunity. Nature medicine 22(1):64-71.

60. Magupalli VG, et al. (2020) HDAC6 mediates an aggresome-like mechanism for NLRP3 and pyrin inflammasome activation. Science 369(6510):eaas8995.

61. Decker $\mathrm{M}$, et al. (2011) Limiting amounts of centrosome material set centrosome size in C. elegans embryos. Current biology : CB 21(15):1259-1267.

62. Pleiner $T$, et al. (2015) Nanobodies: site-specific labeling for super-resolution imaging, rapid epitope-mapping and native protein complex isolation. elife 4:e11349.

63. Edelstein A, Amodaj N, Hoover K, Vale R, \& Stuurman N (2010) Computer Control of Microscopes Using 4 Manager. Current Protocols in Molecular Biology 92(1):14.20.1114.20.17.

64. Edelstein AD, et al. (2014) Advanced methods of microscope control using $\mu$ Manager software. Journal of Biological Methods; Vol 1, No 2 (2014).

65. Olivier N, Keller D, Gönczy P, \& Manley S (2013) Resolution Doubling in 3D-STORM Imaging through Improved Buffers. PLoS One 8(7):e69004.

66. Huang F, et al. (2013) Video-rate nanoscopy using SCMOS camera-specific single-molecule localization algorithms. Nature Methods 10(7):653-658.

67. Wang $Y$, et al. (2014) Localization events-based sample drift correction for localization microscopy with redundant cross-correlation algorithm. Opt. Express 22(13):15982-15991.

68. Ovesny M, Krizek P, Borkovec J, Svindrych Z, \& Hagen GM (2014) ThunderSTORM: a comprehensive ImageJ plug-in for PALM and STORM data analysis and super-resolution imaging. Bioinformatics (Oxford, England) 30(16):2389-2390.

69. Pettersen EF, et al. (2021) UCSF ChimeraX: Structure visualization for researchers, educators, and developers. Protein science : a publication of the Protein Society 30(1):70-82. 